\title{
Mortalidade infantil e gênero no Brasil: uma investigação usando dados em painel
}

\author{
Infant mortality and gender in Brazil: an investigation using \\ updated statistics
}

Taytiellen Fernandes Alves (https://orcid.org/0000-0002-2465-1356) ${ }^{1}$

Alexandre Bragança Coelho (https://orcid.org/0000-0002-9735-7035) ${ }^{1}$

${ }^{1}$ Departamento de Economia Rural, Centro de Ciências Agrárias, Universidade Federal de Viçosa. Av. P. H. Rolfs $\mathrm{s} / \mathrm{n}$, Campus Universitário. 36571-000 Viçosa

MG Brasil.

taytiellen@hotmail.com

\begin{abstract}
Various studies reveal that male mortality is higher than female mortality among children in Brazil. Recent studies have also revealed a reduction in excess male infant mortality in the last few years. However, little is known about which factors are associated with this phenomenon. Since male infants are generally more susceptible, a potential hypothesis is that an improvement in income levels and pre- and post-natal healthcare have a greater impact on reducing male infant mortality. Thus, the scope of this article was to analyze how these factors affect infant mortality in Brazil. For the creation of the model, data for the period from 1996 to 2014 from Brazilian states were utilized. The results indicate that average income, low birth weight, the number of prenatal visits and the fertility rate, are important factors associated with infant mortality in the country. In general, the impact of these variables is greater in the mortality of male children, which would indicate that the higher susceptibility of male children requires greater care from the parents and the health authorities. Furthermore, future studies could analyze the importance of breastfeeding on infant mortality and gender in Brazil, in order to verify the impact of breastfeeding on the reduction of infant deaths.
\end{abstract}

Key words Infant mortality, Gender, Excess mortality
Resumo Diversos estudos indicam que a mortalidade para crianças do sexo masculino é maior do que a do feminino no Brasil. Estudos recentes também têm mostrado uma redução na sobremortalidade infantil masculina nos últimos anos. Entretanto, pouco se sabe sobre quais fatores estão associados a esse fenômeno. Como os bebês do sexo masculino são, em geral, mais frágeis, uma hipótese é de que melhorias no nível de renda e cuidados com a saúde pré e pós-natal tenham um impacto maior na redução da mortalidade infantil masculina. Dessa forma, o objetivo deste artigo foi analisar como esses fatores afetam a mortalidade no Brasil. Para a estimação do modelo, utilizaram-se dados estaduais do Brasil do período de 1996 a 2014. Os resultados indicam que a renda média, o baixo peso ao nascer, o número de consultas de pré-natal e a taxa de fecundidade são importantes fatores associados à mortalidade infantil no país. De modo geral, o impacto dessas variáveis é maior na mortalidade de crianças do sexo masculino, evidenciando que a maior fragilidade dessas crianças exige maior cuidado de pais e autoridades de saúde. Além disso, outros estudos podem analisar a importância do aleitamento materno sobre a mortalidade infantil e gênero no Brasil, de forma a verificar o impacto da amamentação na redução dos óbitos infantis.

Palavras-chave Mortalidade infantil, Gênero, Sobremortalidade 


\section{Introdução}

A taxa de mortalidade infantil é um dos indicadores frequentemente usados para avaliar as condições de vida de uma sociedade. Ela é, em geral, calculada pelo número de crianças que morrem antes de completar um ano de vida a cada mil nascidas vivas. As principais causas de óbito nessa idade estão associadas à prematuridade, à doença diarreica, às anomalias congênitas, à asfixia no parto, à sepse neonatal e à desnutrição ${ }^{1}$.

De acordo com França et al. ${ }^{1}$, a maior parte desses óbitos se concentra no primeiro mês de vida e a elevada participação das causas perinatais, como a prematuridade, evidencia a importância dos fatores ligados à gestação, ao parto e ao pós-parto, em geral passíveis de serem prevenidas por meio de assistência à saúde de qualidade. Outros fatores, como a desnutrição e a diarreia, estão associados às condições de vida da família, como renda insuficiente, falta de saneamento básico, baixa escolaridade, ausência de cuidados básicos no sistema de saúde. Além desses fatores, há ainda ausência de aleitamento materno exclusivo até os seis meses de idade com introdução alimentar nesse período e ausência de treinamento educativo de apoio à amamentação nas Unidades Básicas de Saúde ${ }^{2,3}$.

Nos primeiros meses de vida, a proteção concedida pelo aleitamento materno é maior contra a mortalidade infantil. Porém, em 2006, entre dois e três meses de idade, $56,8 \%$ das crianças já alimentavam-se com mingaus, quase $40 \%$ já consumiam sucos e $7,8 \%$ consumiam comida de sal, colocando a saúde das crianças em maior risco devido à falta de aleitamento materno exclusivo e à introdução inadequada de alimentos. Além disso, a Unidade Básica de Saúde tem como objetivo a promoção da saúde e a redução da mortalidade infantil, mas muitas vezes há falta de sensibilização e capacitação dos profissionais de saúde para orientar as mães, o que contribui para o aumento da mortalidade infantil ${ }^{2-3}$.

Diversas pesquisas evidenciam maior taxa de mortalidade para crianças do sexo masculino: dados do IBGE ${ }^{4}$, por exemplo, mostram que em 2015, no Brasil, a probabilidade de crianças do sexo masculino não completarem o primeiro ano de vida é de 14,9 por mil nascidos vivos e para o sexo feminino, de 12,7 por mil nascidos vivos. As causas dessa diferença estão ligadas a fatores biológicos, que indicam uma maior fragilidade de bebês do sexo masculino a alguns tipos de doença ligados a causas externas, como diarreia, hemorragias e pneumonia. Os fetos masculinos apresentam maior risco de abortamento devido a maior incidência de alterações genéticas.

Outras causas externas também podem influenciar a taxa de mortalidade. Diversos estudos apontam que estresse durante a gravidez aumenta a probabilidade de reduzir a razão entre homens e mulheres, pois mães expostas a maiores níveis de estresse tiveram menor número de filhos do sexo masculino. Por outro lado, em países em desenvolvimento, crianças do sexo masculino estão na maioria das vezes associadas a maiores retornos financeiros no futuro e por este motivo elas podem ter prioridades ao acesso a alimentação de qualidade e cuidados de saúde 5 .

Nos últimos anos, o Brasil ganhou destaque no cenário internacional pelo fato de ter conseguido uma grande redução na taxa de mortalidade infantil. Em 2000, a taxa de mortalidade de crianças era de 29,0 por mil nascidos vivos; já em 2010, passou para 17,22 e em 2015 caiu para 13,8 óbitos por mil nascidos vivos4. Mesmo diante deste declínio observado nos últimos anos, o país ainda apresenta altas taxas de mortalidade quando comparado a países desenvolvidos. Segundo França e Lansky ${ }^{6}$, a taxa de mortalidade infantil brasileira ainda é cerca de 3 a 6 vezes maior do que a de países como Japão.

Além disso, ainda há grande heterogeneidade regional no país. Em 2015, a menor taxa de mortalidade infantil brasileira encontrava-se no Espírito Santo, com 9,2 óbitos para cada mil nascidos vivos; já a maior taxa estava no Amapá, com 23,5 óbitos por mil nascidos vivos. Em relação às regiões, Mombelli et al. ${ }^{7}$ destacam maiores taxas de mortalidade infantil nas regiões Norte, Nordeste e Centro-Oeste.

Estudos recentes também têm mostrado uma redução na sobremortalidade infantil masculina nos últimos anos ${ }^{8}$. Entretanto, pouco se sabe sobre quais fatores estão associados a esse fenômeno. Como os bebês do sexo masculino são, em geral, mais frágeis, uma hipótese é de que melhorias no nível de renda e cuidados com a saúde pré e pós-natal tenham um impacto maior na redução da mortalidade infantil masculina. Nesse contexto, o objetivo deste trabalho é analisar como os fatores socioeconômicos e os indicadores de saúde se relacionam com a taxa de mortalidade infantil por gênero no país, de forma a contribuir com a literatura brasileira no que refere-se as causas da mortalidade infantil por gênero, além de analisar separadamente o efeito da renda sobre a mortalidade infantil. 


\section{Metodologia}

Para investigar a mortalidade infantil, gênero e renda no Brasil utiliza-se um painel composto pelos 26 estados brasileiros mais o Distrito Federal, para o período de 1996 a 2014, sendo o último ano o mais recente disponível para algumas variáveis utilizadas neste estudo. De acordo com Gujarati e Porter ${ }^{9}$, os dados em painel possuem várias vantagens em relação às séries temporais, pois levam em consideração a heterogeneidade, oferecem dados mais informativos, menos colinearidade entre as variáveis, mais graus de liberdade e eficiência, entre outras vantagens.

Como controle, os modelos incluem, além da renda média estadual, as variáveis "baixo peso ao nascer”, "doenças infecciosas e parasitárias", consulta pré-natal e fecundidade, descritas abaixo. Essas variáveis buscam captar as características socioeconômicas das famílias, além dos cuidados com a saúde. Utiliza-se a estimação por efeitos fixos, que leva em conta as características individuais de cada ano e estado. Para estimação do modelo, houve transformação logarítmica em todas as variáveis.

A especificação dos modelos são:

$$
\begin{aligned}
& \text { TMIfemin }_{i t}=\beta_{1 i}+\beta_{2} \log \text { Rend }_{i t}+u_{i t} \\
& \text { TMImasc }_{i t}=\beta_{1 i}+\beta_{2} \log \text { Renda }_{i t}+u_{i t} \\
& \text { TMIfemin. }{ }_{i t}= \\
& \beta_{1 i}+\beta_{2} \log \text { Renda }_{i t}+\beta_{3} \log \text { fecundidade }+ \\
& \beta_{4} \text { logbaixopeso }+\beta_{5} \text { logpré-natal }+ \\
& \beta_{6} \log \text { doençasinfecciosasparasitárias }+u_{i t} \\
& \text { TMImasc. }_{\text {it }}= \\
& \beta_{1 i}+\beta_{2} \log \text { Rend }_{i t}+\beta_{3} \log f e c u n d i d a d e+ \\
& \beta_{4} \text { logbaixopeso }+\beta_{5} \log \text { pré-natal }+ \\
& \beta_{6} \operatorname{logdoençasinfecciosasparasitárias~}+u_{i t}
\end{aligned}
$$

Em que TMI masc. é a taxa de mortalidade infantil (TMI) para crianças do sexo masculino e TMI femin. é a taxa de mortalidade infantil para crianças do sexo feminino. $\mathrm{O}$ subscrito $t$ indica tempo (período de 1996 a 2014), $i$ indica os Estados brasileiros e Uit é o termo de erro.

Os dados são provenientes do Departamento de Informática do SUS (DATASUS) e do Instituto de Pesquisa Econômica Aplicada (IPEADATA) para os 26 estados brasileiros e o Distrito Federal, com periodicidade anual, no período de 1996 a 2014 (19 anos). Encontram-se no Quadro 1 as variáveis utilizadas, a descrição de cada uma, o sinal esperado (positivo ou negativo), ou seja, a influência de cada variável sobre a taxa de mortalidade infantil e a fonte de dados.

$\mathrm{Na}$ Tabela 1 encontram-se as estatísticas descritivas das variáveis utilizadas: há um total de 513 observações (27 unidades de federação vezes 19 anos) para todas as variáveis, exceto para 'renda', para o qual o número de observações foi inferior devido à ausência de informações dessa variável em alguns anos do período analisado.

Como descrição geral da amostra, a Tabela 1 apresenta o resumo estatístico das variáveis. Percebe-se através da tabela que a média da taxa de mortalidade infantil masculina é maior do que a média da mortalidade de crianças do sexo feminino, 20,4 e 16,6 respectivamente. As crianças da amostra pesam em média 545 gramas e o número médio de mortes de crianças com doenças infecciosas e parasitárias é de 146.

Ainda de acordo com a Tabela 1 , a renda média é de $\mathrm{R} \$ 1.114,00$ e sobre a fecundidade, as mulheres têm em média 6 filhos ao final de seu período reprodutivo. Sobre a variável consulta ao pré-natal, o número médio é de 11 consultas. $\mathrm{Na}$ seção 3, encontram-se os resultados obtidos através da estimação do modelo.

\section{Resultados e discussão}

Nas colunas 1 e 2 da Tabela 2, encontram-se os resultados do modelo estimado para taxa de mortalidade infantil feminina e taxa de mortalidade infantil masculina, respectivamente, tendo como variável explicativa apenas a renda. Os resultados para estes modelos foram estatisticamente significativos, indicando que a renda tem expressivo efeito sobre a mortalidade infantil por gênero no Brasil. Como visto na coluna 2, aumentos na renda reduz mais a mortalidade infantil masculina.

Bezerra Filho et al..$^{10}$ encontraram resultados análogos, em que aumento na renda foi importante causa para redução da mortalidade infantil e enfatizam a importância na melhoria de fatores socioeconômicos, uma vez que melhor distribuição de renda mostrou-se decisiva para a sobrevivência no primeiro ano de vida.

Nos modelos estimados das colunas 3 e 4 são incluídas as outras variáveis explicativas além da renda. É possível perceber nestes modelos que mesmo com a inserção de mais variáveis que podem causar a mortalidade infantil, a renda continua com significativo efeito sobre as taxas de mortalidade, apresentando novamente maior impacto sobre a taxa de mortalidade infantil masculina. 
Quadro 1. Descrição dos dados.

\begin{tabular}{|l|l|l|l|}
\hline \multicolumn{1}{|c|}{ Variáveis } & \multicolumn{1}{|c|}{ Descrição } & $\begin{array}{c}\text { Sinal esperado } \\
\text { (efeito das variáveis } \\
\text { sobre a TMI) }\end{array}$ & \multicolumn{1}{|c|}{ Fonte de dados } \\
\hline $\begin{array}{l}\text { Taxa de Mortalidade } \\
\text { Infantil }\end{array}$ & $\begin{array}{l}\text { Óbitos de crianças menores de um ano de } \\
\text { idade por mil nascidos vivos }\end{array}$ & Datasus \\
\hline Renda & $\begin{array}{l}\text { Renda média - soma da renda de todos os } \\
\text { trabalhos }\end{array}$ & Negativo & Ipeadata \\
\hline Fecundidade & $\begin{array}{l}\text { Número médio de filhos que uma mulher } \\
\text { teria ao fim de seu período reprodutivo }\end{array}$ & Positivo & Datasus \\
\hline Baixo peso ao nascer & $\begin{array}{l}\text { Número médio anual de crianças com } \\
\text { baixo peso ao nascer }\end{array}$ & Positivo & Datasus \\
\hline Consulta pré-natal & Número médio de consultas pré-natal & Negativo & Datasus \\
\hline $\begin{array}{l}\text { Doenças infecciosas } \\
\text { e parasitárias }\end{array}$ & $\begin{array}{l}\text { Número médio anual de crianças com } \\
\text { doenças infecciosas e parasitárias ao nascer }\end{array}$ & Positivo & Datasus \\
\hline
\end{tabular}

Fonte: Elaboração Própria.

Tabela 1. Estatísticas descritivas.

\begin{tabular}{lcrr}
\hline \multicolumn{1}{c}{ Variáveis } & $\begin{array}{c}\text { Número de } \\
\text { observações }\end{array}$ & Média & Desvio Padrão \\
\hline Taxa de Mortalidade Infantil Feminina & 513 & 16,600 & 4,799 \\
Taxa de Mortalidade Infantil Masculina & 513 & 20,365 & 6,284 \\
Baixo peso & 513 & 545,517 & 639,393 \\
Renda & 459 & $1.144,036$ & 433,836 \\
Doenças infecciosas e parasitárias & 513 & 146,618 & 180,685 \\
Consulta pré natal & 513 & 11,069 & 0,974 \\
Fecundidade & 513 & 6,317 & 0,944 \\
\hline
\end{tabular}

Fonte: Resultados da pesquisa obtidos a partir dos dados do DATASUS E IPEADATA.

Tabela 2. Resultados dos modelos estimados.

\begin{tabular}{lcccc}
\hline \multicolumn{1}{c}{ Variáveis } & TMI femin. & TMI masc. & TMI femin. & TMI masc. \\
& $(\mathbf{1})$ & $(\mathbf{2})$ & $(\mathbf{3})$ & $-14.550^{\star}$ \\
\hline Renda & $-10.005^{*}$ & $-13.419^{*}$ & $-10.814^{*}$ & $(2.934)$ \\
& $(0.798)$ & $(1.016)$ & $(2.323)$ & $0.135^{\star *}$ \\
Fecundidade & & & $0.111^{\star *}$ & $(0.054)$ \\
& & & $(0.043)$ & $0.004^{* *}$ \\
Baixo peso & & $0.771^{\text {ns }}$ & $(0.001)$ \\
ao nascer & & $(1.033)$ & $-6.477^{\star}$ \\
Pré-natal & & $-5.305^{* *}$ & $(2.829)$ \\
& & & $(2.458)$ & $0.594^{\text {ns }}$ \\
Doenças infecciosas e parasitárias & & & $0.655^{\star * *}$ & $(0.431)$ \\
& & & $(0.334)$ & Sim \\
Efeito fixo de ano & Sim & Sim & Sim & Sim \\
Efeito fixo de Estado & Sim & Sim & Sim &
\end{tabular}

Fonte: Resultados da pesquisa obtidos a partir dos dados do DATASUS E IPEADATA.

Nota: Erros padrão robustos estão entre parênteses. $\left({ }^{*}\right)$ denota significância a $1 \%,\left({ }^{* *}\right)$ denota significância a $5 \%,\left({ }^{* *}\right)$ denota significância a $10 \%$ e (ns) não significativo. Regressões robustas à heterocedasticidade. 
Garcia e Santana ${ }^{11}$ estudaram a evolução da desigualdade socioeconômica na mortalidade infantil no Brasil e chegaram a resultados semelhantes ao deste estudo, constatando concentração dos óbitos infantis cujas mães tinham menor renda per capita. Estes resultados evidenciam que as crianças do sexo masculino são mais sensíveis a alterações na renda do que as do sexo feminino, uma vez que o aumento na renda reduz mais a mortalidade das crianças do sexo masculino. Menezes et al. ${ }^{12}$ estudaram a mortalidade infantil em Pelotas e constaram maior risco de mortalidade para os meninos, no qual o coeficiente de mortalidade infantil apresentou-se duas vezes maior para o sexo masculino do que para o sexo feminino. Szwarcwald et al. ${ }^{13}$ também constaram menor esperança de vida ao nascer para crianças do sexo masculino.

A variável fecundidade apresentou sinal de acordo com o esperado e foi estatisticamente significativa em todos os modelos analisados. Percebe-se com isso, sua importância na mortalidade infantil por gênero. Para Paixão e Ferreira ${ }^{14}$, redução da fecundidade reduz também a TMI, de modo análogo, quando há aumento da fecundidade há aumento da TMI, pois quanto maior o número de filhos menor a quantidade de tempo que as mães podem dedicar-se ao cuidado com cada um deles e consequentemente menor expectativa de vida.

Sobre a variável baixo peso ao nascer, apareceu com o sinal de acordo com o esperado em todos os modelos analisados, mas não foi estatisticamente significativa para a mortalidade infantil feminina (coluna 3). Geib et al. ${ }^{15}$ e Menezes et al. ${ }^{12}$ encontraram resultados análogos.

O número médio de consultas pré-natal apresentou sinal de acordo com o esperado em todos os modelos analisados e foi estatisticamente significativo. Os resultados indicam que o acesso ao pré-natal é importante para reduzir a taxa de mortalidade infantil, principalmente para as crianças do sexo masculino, pois quando há aumento no número de consultas pré-natal reduz-se mais a mortalidade de crianças do sexo masculino. No Brasil houve avanços na cobertura ao pré-natal, mas a qualidade ainda é precária. A literatura mostra relação entre maior número de consultas pré-natal e redução da mortalidade infantil no primeiro ano de vida. $\mathrm{O}$ fato de grande parte dos óbitos ocorrerem no primeiro ano de vida enfatiza a importância dos cuidados durante a gestação para a redução da mortalidade infantil ${ }^{1-16}$.

Para Careti et al. ${ }^{17}$, há maior concentração de óbitos de crianças de até um ano na população de baixa renda, pois as mães que compõem esta população não conseguem realizar devidamente o pré-natal e o óbito das crianças com menos de uma semana de vida é mais frequente do que o óbito daquelas com idade mais avançada. Com isso, percebe-se a influência da renda no acesso ao pré-natal e, consequentemente, na mortalidade infantil.

Em relação à variável doenças infecciosas e parasitárias, ela foi estatisticamente significativa apenas para crianças do sexo feminino. De acordo com Buchalla et al. ${ }^{18}$ as causas de mortalidade infantil por doenças infecciosas têm reduzido muito nos últimos anos, tornando-se muito baixa, o que pode explicar a não significância estatística dessa variável para os outros modelos estimados.

Além das variáveis utilizadas neste trabalho, outros fatores podem contribuir para a redução da taxa de mortalidade infantil, como o aleitamento materno exclusivo até os seis meses de idade, que previne também a desnutrição e o desmame precoce. A introdução de outros alimentos antes dos seis meses de idade pode acarretar em elevado risco de alimentos contaminados pela administração inadequada, provocando aumento da mortalidade infantil. Além disso, é de suma importância a atuação do enfermeiro, juntamente com a Unidade Básica de Saúde nesse período de orientação à mãe ${ }^{19}$.

\section{Conclusão}

O trabalho contribui com a comunidade científica no que refere-se à taxa de mortalidade infantil por gênero. Os resultados mostram que melhorias no nível de renda e cuidados com a saúde pré e pós-natal têm um impacto maior na redução da mortalidade infantil masculina.

A fragilidade de bebês do sexo masculino deveria implicar em políticas de saúde específicas, que levassem em consideração o gênero da criança. O governo deve, portanto, dar ênfase às gestantes de crianças do sexo masculino, com o intuito de reduzir a taxa de mortalidade destas, as quais dentre as variáveis analisadas mostraramse mais suscetíveis a óbito.

Além disso, é importante maior investimento na Unidade Básica de Saúde, com a atuação de grupos de educação para a saúde, capacitando as equipes para que haja maior incentivo às mães para o aleitamento materno exclusivo e, consequentemente, redução da mortalidade infantil. Dessa forma, outros estudos podem analisar a 
importância do aleitamento materno sobre a mortalidade infantil e gênero no Brasil, de forma a verificar a influência do desmame precoce nos óbitos infantis.

\section{Colaboradores}

TF Alves e AB Coelho trabalharam em conjunto para realização do trabalho.

\section{Agradecimentos}

$\mathrm{O}$ autor Alexandre Bragança Coelho agradece o apoio financeiro do CNPq, por meio de Bolsa de Produtividade em Pesquisa.

\section{Referências}

1. França EB, Lansky S, Rego MAS, Malta DC, França JS, Teixeira R, Porto D, Almeida MF, Souza MFM, Szwarcwald CL, Mooney M, Naghavi M, Vasconcelos AM. Principais causas da mortalidade na infância no Brasil, em 1990 e 2015: estimativas do estudo de carga global de doença. Rev Bras Epidemiol 2017; 20(1):4660 .

2. Corrêa AMS, León LM, Panigassi G, Rea MF, Escamilla RP. Amamentação e alimentação infantil. In: Brasil. Ministério da Saúde (MS). Pesquisa Nacional de Demografia e Saúde da Criança e da Mulher 2006. Brasília: PNDS; 2009. p. 1-302.

3. Bezerra LCA, Frias PG, Vidal SA, Macedo VC, Vanderlei LC. Aleitamento materno: avaliação da implantação do programa em unidades básicas de saúde do Recife, Pernambuco (2002). Cien Saude Colet 2007; 12(5):309-317.

4. Instituto brasileiro de geografia e estatística (IBGE). Tábua completa de mortalidade para o Brasil, 2015. Rio de Janeiro: IBGE; 2016.

5. Filho ADPC, Laurenti R. O sexo masculino vulnerável: razão de masculinidade entre os óbitos fetais brasileiros. Cad Saude Publica 2012; 28(4):720-728.

6. França E, Lansky S. Mortalidade infantil neonatal no Brasil: situação, tendências e perspectivas. In: Anais do XVI Encontro Nacional de Estudos Populacionais; 2008; Caxambú. p. 1-29.

7. Mombelli MA, Sass A, Molena CAF, Téston EF, Marcon SS. Fatores de risco para mortalidade infantil em municípios do estado do Paraná, de 1997 a 2008. Rev Paul Pediatr 2012; 30(2):187-194.

8. Wong LLR, Barros JVS, Boifácio GMO, Braga LJS. Padrões de diferencial por sexo da mortalidade nas primeiras idades: uma investigação com base nas causas de morte. In: Anais doXIX Encontro Nacional de Estudos Populacionais; 2014; São Paulo. p. 1-21.

9. Gujarati DN, Porter DC. Econometria básica. Porto Alegre: E. AMGH; 2011.

10. Bezerra Filho JG, Kerr LRFS, Miná DL, Barreto ML. Distribuição espacial da taxa de mortalidade infantil e principais determinantes no Ceará, Brasil, no período 2000-2002. Cad Saude Publica 2007; 23(5):1173-1185
11. Garcia LP, Santana LR. Evolução das desigualdades socioeconômicas na mortalidade infantil no Brasil, 1993-2008. Cien Saude Colet 2011; 16(9):3717-3728.

12. Menezes AMB, Victora CG, Barros FC, Albernaz E, Menezes FS, Jannke HA, Alves C, Rocha C. Mortalidade infantil em duas coortes de base populacional no Sul do Brasil: tendências e diferenciais. Cad Saude Publica 1996; 12(1):79-86.

13. Szwarcwald CL, Bastos FI, Esteves MAP, Andrade CLT, Paez MS, Medici EV, Derrico M. Desigualdade de renda e situação de saúde: o caso do Rio de Janeiro. Cad Saude Publica 1999; 15(1):15-28.

14. Paixão N, Ferreira T. Determinantes da mortalidade infantil no Brasil. Informe Gepec 2012; 16(2):6-20.

15. Geib LTC, Fréu CM, Brandão M, Nunes ML. Determinantes sociais e biológicos da mortalidade infantil em coorte de base populacional em Passo Fundo, Rio Grande do Sul. Cien Saude Colet 2010; 15(2):363-370.

16. Barbosa T, Coelho KR, Andrade GN, Bittencourt DAS, Leal MC, Gazzinelli A. Determinantes da mortalidade infantil em municípios do Vale do Jequitinhonha, Minas Gerais, Brasil. Rev Min Enferm 2014; 18(4):907-914.

17. Careti CM, Scarpelini AHP, Furtado MCC. Perfil da mortalidade infantil a partir da investigação de óbitos, Rev Eletr Enf 2014; 16(2):352-360.

18. Buchalla CM, Eliseu AW, Laurenti RA. Mortalidade por doenças infecciosas no início e no final do século XX no município de São Paulo. Ver Bras Epidemiol 2003; 6(4):335-344.

19. Amorim MM, Andrade ER. Atuação do Enfermeiro no PSF sobre aleitamento materno. Perspectivas Online 2009; 3(9):93-110.

Artigo apresentado em 28/11/2018 Aprovado em 05/06/2019

Versão final apresentada em 07/06/2019

Editores-chefes: Romeu Gomes, Antônio Augusto Moura da Silva 